\title{
ThermoCam: Smart Baby Monitoring Assistant
}

\author{
M. Eren Akbiyik* ${ }^{*}$, Cagan S. Coban ${ }^{\dagger} \|$, Elif Aygun ${ }^{\ddagger}$, \\ Huseyin Ziya Imamoglu ${ }^{\S} \|$, Doga Gurgunoglu ${ }^{\ddagger}$ and Duygu Ider ${ }^{\mathbb{T}}$ \\ ${ }^{*}$ IBM Deutschland GmbH, Böblingen, Germany ${ }^{\dagger}$ Koc University, Istanbul, Turkey

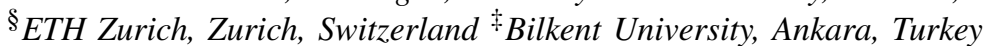 \\ II Humboldt University, Berlin, Germany \\ *erenakbiyik@gmail.com, ${ }^{\dagger}$ ccoban20@ku.edu.tr, ${ }^{\sharp}\{$ elif,gurgunoglu\}@ee.bilkent.edu.tr, \\ §zimamoglu@student.ethz.ch, IIiderduyg@hu-berlin.de
}

\begin{abstract}
Fever is one of the main symptoms of various illnesses during infancy and it requires continuous supervision especially above 38 degree Celsius as fatal complications may develop. With the emerging IoT and e-Health technologies, a variety of different consumer products are being developed to facilitate the fever monitoring for parents. In this study, our aim is to develop a prototype that will continuously track the body temperature of an infant using an RGB and a thermal camera, and provide different notification capabilities for the users. The product is expected to work effectively from 1-2 meters of distance with approximately 0.4 degree Celsius accuracy. The real-time video display is included to the system with one second of latency for video, audio and alarm features. The user interface also provides room temperature and humidity data, along with measured statistics of baby body temperature. The system is also able to alarm the user upon the detection of loud noise in the environment, which can indicate interruptions in baby's sleep. The prototype is expected to provide an affordable, all-in-one solution for the parents as a baby monitoring device.
\end{abstract}

Keywords-Baby monitor, home monitoring, thermal imaging, eHealth, IoT

\section{INTRODUCTION}

Fever, mostly an indicator of infectious diseases, is considered a dangerous physiological response for infants, especially for neonates who require immediate hospitalization [1]. Neonatal age requires constant and sensitive monitoring of body temperature because fever is prone to be a dangerous symptom depending on how long it lasts, and for especially premature infants, it may lead to the development of fatal conditions as they do not have an adaptive thermo-regulation mechanism [1], [2]. Hence, fever monitoring plays an important role in the challenging course of early parenthood.

The proposed Smart Baby Monitoring Assistant (ThermoCam) is designed to facilitate infant fever monitoring and address the insufficiency of temperature-tracking baby monitors in the national and global markets. Existing baby monitors require the use of external hardware to measure temperature and the excessive cost of the current products for domestic use. Based on the conducted market research, ThermoCam is a unique product, because it is a reliable

\footnotetext{
$\|_{\text {Equal contribution }}$
}

and safe baby monitor that offers an easy-to-use interface to parents [3]-[6]. Considering the risks of fever for infants, this product bears great significance for users in the global market.

The proposed design is built on a low-cost system, which is able to measure the body temperature of the baby and report the data to the end user in real-time through a web interface. Since it is a proof-of-concept device, ThermoCam does not ensure a medical-level accuracy. The system is built to detect fluctuations in body temperature and determine whether this change leaves the normal temperature range, which is determined to be between $36.5-38^{\circ} \mathrm{C}$ by American Academy of Pediatrics/American Congress of Obstetricians and Gynecologists [7].

The system consists of a Raspberry Pi 3B+ device, a RGB camera and a thermal camera. The video data from the two cameras are overlaid in order to measure body temperature from the face of the infant using face detection methods [8]. The maximum point in the region of interest is taken as the body temperature and its change over time is monitored through the algorithm.

\section{RELATED WORK}

In the global market, real-time video streaming, room temperature and humidity tracking, and two-way talking are typical properties of baby monitors. Most products also have infrared night vision in order to provide night-time as well as day-time video [3], [4]. Some monitors consist of a camera setup that is controlled by an additional device with a screen, whereas others are tracked using a smartphone application instead [5].

There are baby monitors that make use of thermal data for purposes besides providing night vision. For example, a monitor allows the users to define a region of interest and notifies them in case the target leaves this region, using the thermal data obtained from FLIR ONE [9]. There are some other products that track body temperature using an electronic device that is connected to the target's body [6], [10].

Smart Baby Monitoring Assistant is an integration of two types of baby monitors in the market: Ones that provide 
video streaming from an additional device, and others that track body temperature using wearables and deliver to parents through a website. However, ThermoCam eliminates the risks associated with these two types of monitors, as it measures body temperature from a distance of 1-2 meters, and therefore is more reliable compared to wearable that can accidentally be detached from the target's body and eliminates the risk of ingestion by the baby. Additionally, ThermoCam's website interface facilitates usage by eliminating the need of an additional device. Therefore, ThermoCam is a unique baby monitor in the global market. However, when we go out of the scope of the baby monitors, there were similar devices on the market such as Avio TVS200IS [11]. This particular device could fuse thermal and RGB videofeed while also outputting a temperature reading with $\pm 1.0 \mathrm{C}^{\circ}$ accuracy. However, this product was geared for professional use in airports for tracking of contagious diseases such as flu or ebola and did not have the sound tracking and live audio/video audiostream properties that ThermoCam has. There is also the possibility that it had a cost that was much higher than ThermoCam considering that similar cameras from the same company have prices reaching up to 2000 [12]. There are also similar systems offered by FLIR such as FLIR A320 for deployment in airports [13]. However these systems are very expensive when compared to the prototype costs of ThermoCam [14].

\section{SySTEM OVERVIEW}

ThermoCam, as shown in Figure 1, consists of a hardware platform based on Raspberry Pi 3B+, and an image processing and video alignment framework programmed in Python. Raspberry $\mathrm{Pi} 3 \mathrm{~B}+$ is used as the main hardware for the proof-of-concept prototype. For peripherals, the system incorporates a FLIR Lepton Thermal Camera [15] coupled with a WaveShare PiCam [16], a humidity and temperature sensor (DHT-22) [17], a USB soundcard (UGREEN USB external sound adapter) [18], generic stereo speakers and a Piranha PRN-202 external laptop microphone [19].

The system fundamentally carries out four main functions. The first function is to continuously monitor the baby's
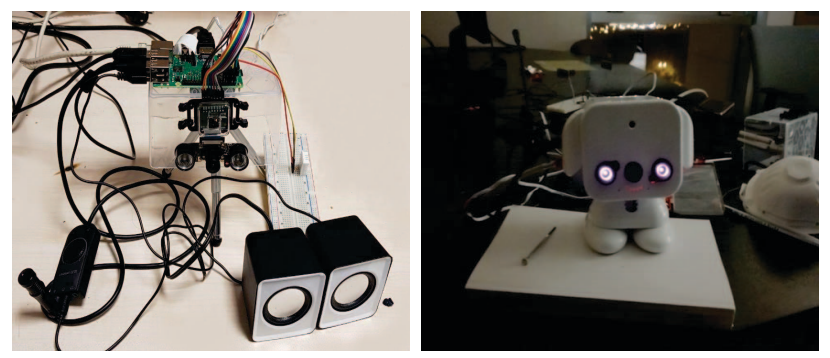

Figure 1. (Left) Initial system and (right) final prototype on low-light conditions. Components of the initial system are boxed into a manageable size, with smaller alternatives chosen for the speakers. Light of IR LEDs is unperceivable to human eye.

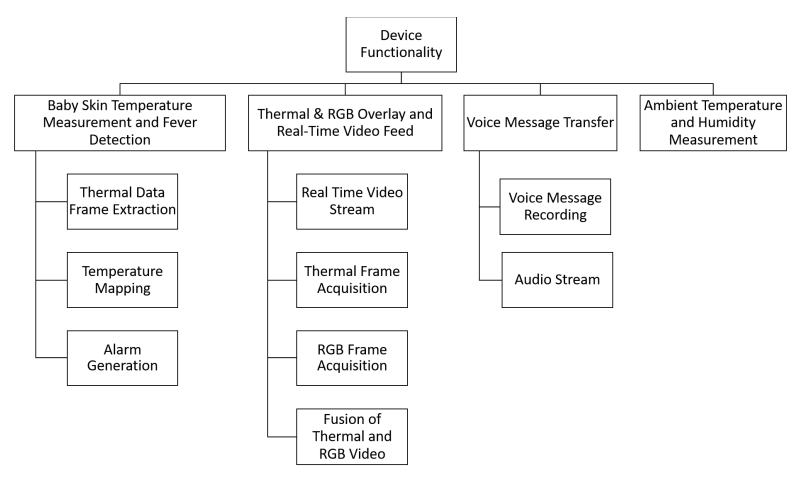

Figure 2. Functionality Diagram

body temperature. This is achieved by establishing a region of interest defined as the baby's face and then taking the maximum temperature in the region. If there is a lifethreatening temperature change in the surface temperature of the baby's face, ThermoCam sends out an alarm to the user's smartphone or computer. The second function of the system is to overlay the thermal and RGB video streams, creating a live video feed that is accessible to the user and delivering up to date information about the temperatures throughout the baby's face. The video feed may also be shown only in RGB, depending on the user's preference. The user can reach the video feed through a website. The third function of the system is to transfer voice messages between the baby and the user, indicating that parents can speak to their babies and listen back using ThermoCam.

The final function of the system is to measure the ambient temperature and humidity of the baby's room. This function is critical, because environmental parameters are crucial for the well-being of the baby and therefore, information about room conditions should be delivered to parents on real-time. Thus, ThermoCam incorporates all functions of the baby monitors currently in the national and global markets, and additionally includes baby temperature measurement tracking, fever detection and real-time thermal-RGB overlaid video feed, as displayed in the functionality diagram of the system in Figure 2.

\section{System ARChitecture}

In this part, the architecture of the system which consists of hardware and software architecture will be explained in detail.

\section{A. Hardware Architecture}

The hardware architecture mainly consists of four parts, development board, image acquisition hardware, sound playback and acquisition hardware, and ambient data acquisition hardware. A diagram to visualize the connections between the components can be seen in Figure 3. 


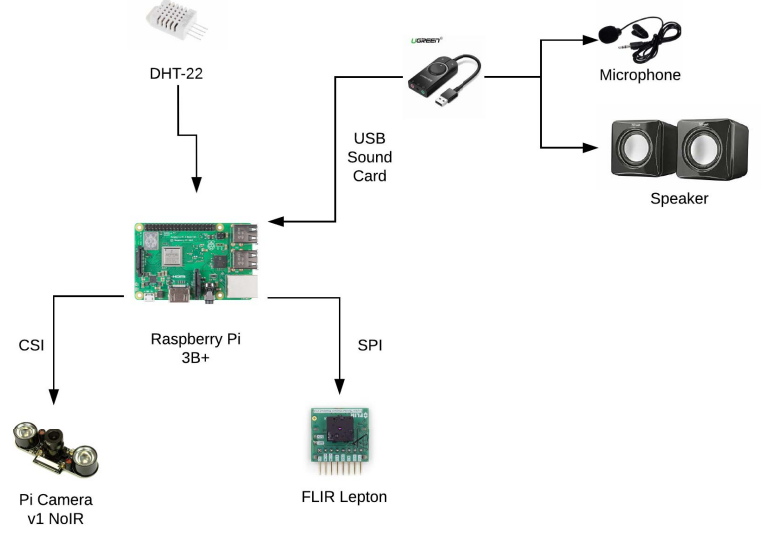

Figure 3. Hardware Architecture

1) Development Board: Raspberry Pi $3 \mathrm{~B}+$ is selected as the development board for this system. It is responsible for running the software framework, handling peripherals and connecting to the server for data transfer. Moreover, Raspberry $\mathrm{Pi}$ is chosen as the main hardware of the system as it's comparably cheap, has community support and thus perfect for prototyping purposes.

2) Image Acquisition Hardware: This hardware consists of the thermal camera and the RGB camera employed in the system. The RGB camera is a Waveshare v1.0 no-IR camera 5MP. The camera includes two on board IR LEDs and can record $1080 \mathrm{p}$ video at $30 \mathrm{FPS}$. The model of the thermal camera is FLIR Lepton 1.5, with resolution $80 x 60$. This model lacks the radiometry option, consequently requires a mapping from pixel temperature values to the real temperature. The camera can output a video from capturing a sequence of images at an average of 8.7 FPS.

3) Sound Playback and Acquisition Hardware: This hardware includes the UGREEN external sound card, Piranha PRN-202 external laptop microphone and generic USB-powered stereo speakers. The microphone can work effectively up to 2 meters, coinciding with the anticipated working distance of the device.

4) Ambient Data Acquisition Hardware: This hardware consists of Adafruit DHT-22 temperature and humidity sensor. This sensor can output humidity readings with an accuracy of $2-5 \%$ and output temperature readings with $\pm 0.5{ }^{\circ} \mathrm{C}$ accuracy.

\section{B. Software Architecture}

1) Device: Raspbian version 2018-11-13 is used on Raspberry $\mathrm{Pi}$ as the operating system and source code is run on Python 3 programming language. The source code is based on a multiprocessing architecture that handles multiple device operations such as image [20] and audio processing, server communication and baby action detection concurrently.
Four physical core of the processor need to be used in parallel to satisfy the design requirements.

Multiprocessing architecture consist of 4 main processes that are named as control_routine, video_routine, audio_routine and server_routine. A data structure similar to dequeue is implemented over Queue structure of multiprocessing package, to ensure that device provides real-time data in case of a latency. The overriding version is designed to serve storage for video frames and audio by dumping the last object to free a slot for the incoming data instead of giving an exception when user tries to put a new object to a full queue. The visualization between the connections of each routine can be seen in Figure 4.

There are distinct roles for each process: video_routine acquires both RGB and thermal video frames and overlays them according to a predetermined color map. audio_routine acquires the audio feed and detects baby cry by frequency analysis over a sliding window of time points and simple thresholding. server_routine parses the data from all other routines and synchronizes them if necessary. Then, it sends the data (temperature, humidity, alarms) to the server by RESTful API, video and audio streams sent by web sockets. For video streaming, the current system uses MJPEG [21] protocol the device serve up to $30 \mathrm{fps}$ with some repeated frames, since thermal camera can provide a maximum of 9 frames-per-second.

2) Multimodal Image Registration: With different positions and focal structures, the alignment of thermal and RGB cameras is a crucial part of the proposed design. Posed as the problem of multimodal image registration in the literature, the main approaches include feature calibration over respective feeds, series of segmentation and alignment to overlay ROI's, and automatic registration of images using neural networks [26], [27].

For the data acquired from the system architecture, nonsimilar visual features of the thermal sensor make it inefficient to use feature detection and matching methods such as ORB, FAST and SIFT [28]; and the lack of labeled visual data prevents the training of complex neural nets to create an acceptable warping. To solve the problem of inconsistent

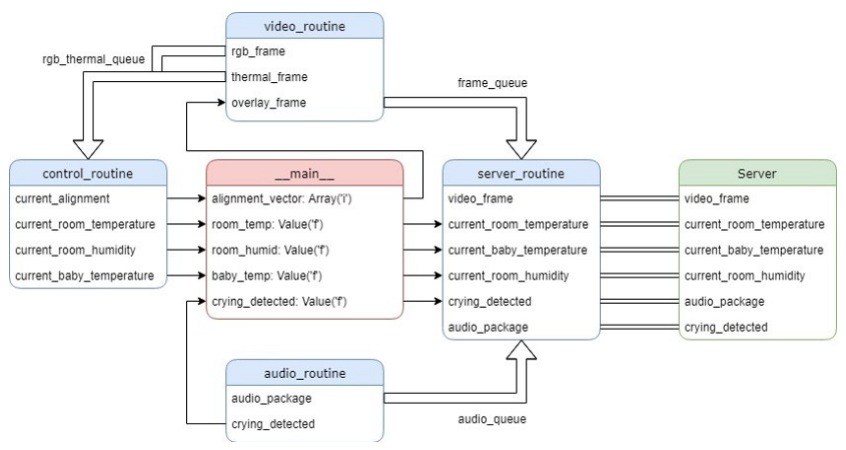

Figure 4. Device Software Flowchart 
features, an algorithm is used from [29] to find the ideal polynomial warping to align the thermal frame on the RGB.

To proceed with the description of the algorithm, we provide two assumptions for a camera-sensor system:

- The edge profile of the sensor is sufficiently informative, i.e. there exists features that correspond to both the edge profiles of the RGB image and the image-like data provided by the thermal sensor.

- The position of these two sources are known (whether they are vertically or horizontally aligned) and their location are sufficiently close

The algorithm that is used to calculate the ideal parameters for the second degree polynomial transformation matrix is listed as Algorithm 1. For the exemplary applications of the proposed algorithm, see Results.

For the coverage parameter in the algorithm, $c=4$ is found to provide the ideal signal-to-noise ratio to calculate the crosscorrelation from edge-like features in both thermal and RGB frames via trial and error. The depth of the algorithm, N, is limited by the smallest informative vertical division of the thermal image, which is empirically determined to be 8 for an ideal estimation.

3) Temperature Mapping: Monitoring system of ThermoCam contains a microbolometer-based thermal camera with a resolution of $60 \times 80$ that maps the temperature into pixel values of 14-bits. The mapping of this thermal sensor is not linear, and to capture the underlying polynomial a series of samples from a calibrated black body is taken using the setup shown in Figure 5. For each sample, the temperature

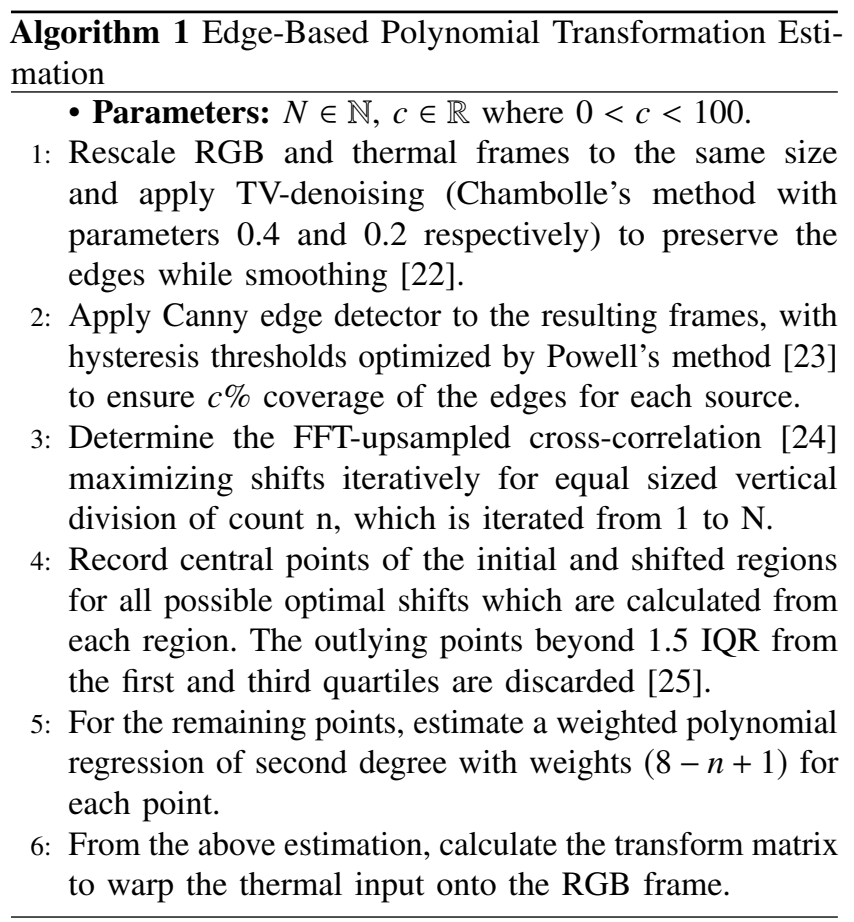

degrees (in Kelvin with a scale of 100) of Focal Plane Array (FPA) and Auxiliary (AUX) sensors are also acquired from the microbolometer via $\mathrm{C}++$ commands over $\mathrm{I}^{2} \mathrm{C}$ bus.

There is no clear instruction regarding to the correlation of FPA and AUX temperatures, which reflect the ambient temperature of the camera for different locations. Therefore, they are used together with the temperature pixel values to create an accurate model. A linear regressor with Elastic Net regularization [30] is cross validated over the dataset, and the ideal first-order model is determined to have only LASSO regularization with zero coefficient for the FPA sensor value.

The resulting relationship between the real temperature $T_{\text {real }}$, pixel value $T_{P}$ and AUX sensor temperature $T_{A U X}$ can be expressed as:

$$
T_{\text {real }}=0.0403 T_{P}+1.2803 T_{A U X}-322.895
$$

The created model is utilized to measure the temperature of the baby over a periodically acquired region of interest via control_routine. RoI is established by a pre-trained Haar Cascade algorithm for face recognition, and a simple blob detection algorithm over thermal camera is employed in the cases that the former algorithm fails, e.g. when encountered with unpredictable postures of the subject.

4) Server: On the server side, a virtual private server is initiated with the Ubuntu 18.04 LTS operating system. The server-side software is coded with Python 3, Flask framework is used together with the Gunicorn WSGI and MySQL database with SQLAlchemy object-relation mapper. A diagram of the database can be seen in Figure 6. The prototyping server specifications are 1 vCPU, 2 GB RAM and 50GB SSD storage. The internet connection speed is up to $1 \mathrm{Gbps}$.

All of the device functionality is accessed through a web interface as shown in the Figure 7. Each user is able to examine baby temperature, room temperature and humidity through descriptive plots and tables. In addition, users are provided valuable information by notifications and alarms for the baby through this web interface. The device can be configured from this panel as well.

For the video stream, the server and the Raspberry Pi implements the MJPEG streaming protocol. In this protocol video stream is implemented through passing encoding video as compressed .jpg images and sending each frame as so. In
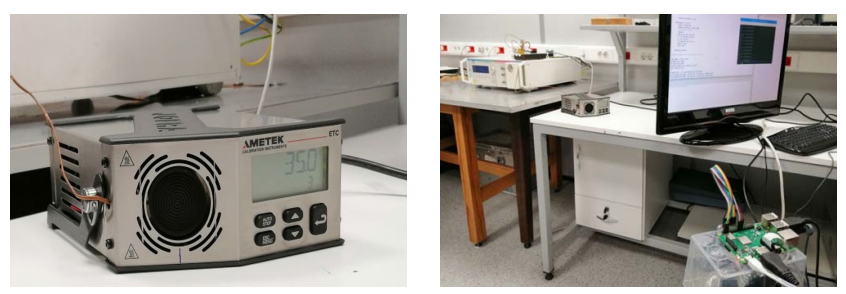

Figure 5. Black body calibration setup 


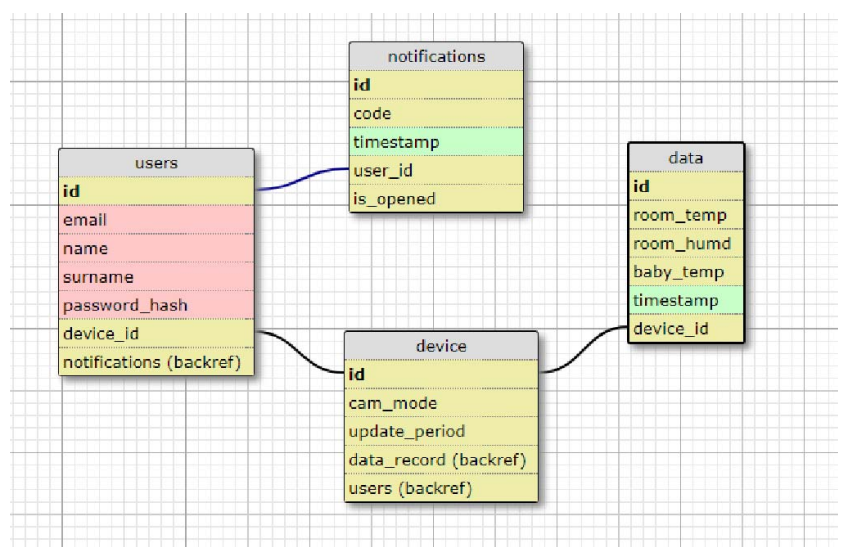

Figure 6. Diagram of the database [31].

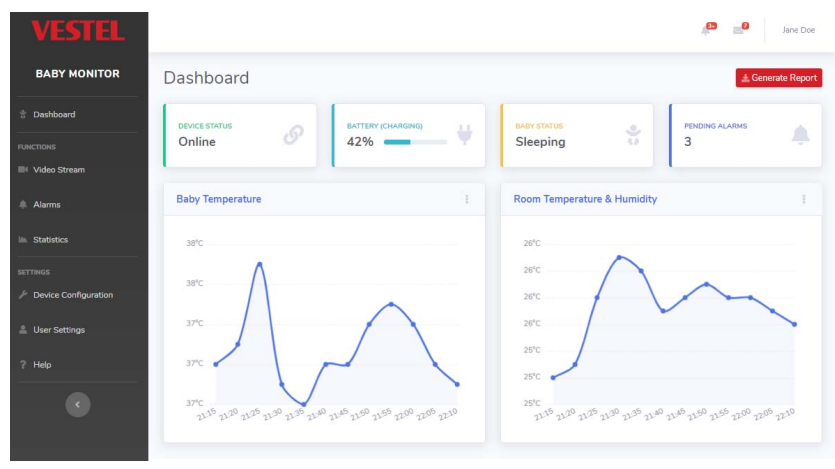

Figure 7. Web Interface

this implementation, we send the size of each frame and the frame itself consequently in a continuous manner.

The database for this project consist of four tables, which are users, notifications, device and data. Each user is linked to a device by device_id foreign key, that is, a device can be accessed by multiple users. The table notifications includes user_id as the foreign key, hence a specific user's notification can be queried. The table data includes device_id as the foreign key to provide link for a device. Each row in this table is a data entry of a device at a specific time instance. This relational model approach gives the capability of querying special properties, for instance user.device.data or user.notification, which enables the web service to work efficiently. The server provides a RESTful web service for CRUD operations. Through this API, the device can record data to the database.

\section{Cost}

The cost associated with the prototype holds vital importance as the cost of the prototype is positively related with the cost of the product. Cost associated with each component of the system is given in Table I in US Dollars. The purchases except for the thermal camera were carried out in Turkey and thus the cost in US Dollars was calculated with the most recent exchange rate which is $1 \mathrm{TL}=0.18 \$$.

The total cost for the prototype is 291.36\$. This is reasonable when we take into account that the system is a proof-of-concept and not a mass production product.

\section{TEST}

Testing the performance of the camera in terms of thermal accuracy is a fundamental step. The test system is designed to reflect real case scenarios that could happen in daily life, as closely as possible. To establish that, a plastic doll of $50 \mathrm{~cm}$ height is purchased and is modified to allow continuous hot water flow with the help of plastic pipes. The water is being heated up with the help of a kettle and pumped into the pipes in to change the surface temperature of the body. The temperature is controlled by an Arduino UNO whose implemented algorithm allows the test system's temperature to alter from $30{ }^{\circ} \mathrm{C}$ and $55{ }^{\circ} \mathrm{C}$. Upper and lower limits are both determined by hardware limitations and project specifications. The diagram of the test system is given in Figures 8.

The aim is to test the device for a singular subject within a range which reflects expected body temperatures of a baby, including fewer, hypothermia and normal conditions, therefore the system is designed to manipulate the surface body temperature from $34^{\circ} \mathrm{C}$ to $38^{\circ} \mathrm{C}$.

The plastic surface is monitored using a thermocouple to assure that the desired surface temperature is reached. Since the plastic has different thermal conduction properties than water, the required time for thermal equilibrium is measured to be more than approximately 10 minutes in our experiments with the help of the Arduino UNO and a waterproof digital thermometer [32], depending on the difference between initial surface temperature and the final

Table I

Component Cost TABle

\begin{tabular}{lc}
\hline Component & Cost (in \$) \\
\hline Raspberry Pi 3B+ & 35 \\
\hline Waveshare Picam 5MP Noir & 27.99 \\
\hline FLIR Lepton Thermal Camera & 184.95 \\
\hline DHT-22 Humidity and Temperature Sensor & 4.52 \\
\hline 32GB microSD Card Class U1 & 6.79 \\
\hline Ugreen USB Soundcard & 12.77 \\
\hline Generic Analog Microphone & 1.83 \\
\hline Generic USB Powered Stereo Speakers & 3.66 \\
\hline Raspberry Pi Power Adapter & 12.82 \\
\hline Miscellaneous Costs (Ribbon cables, jumper & 7.82 \\
\hline Total Cost & $\mathbf{2 9 1 . 3 6}$ \\
\hline
\end{tabular}




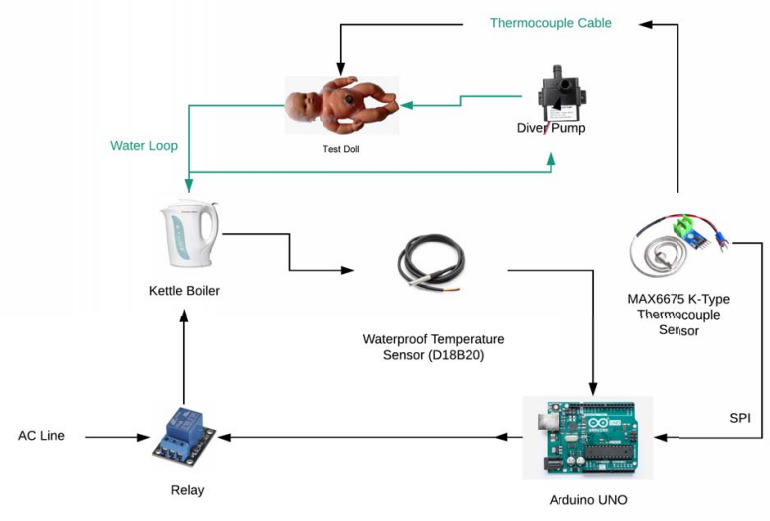

Figure 8 . The test system block diagram

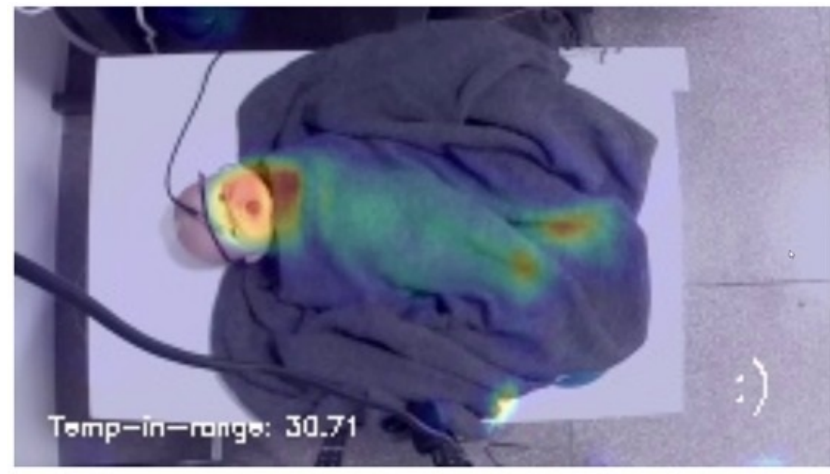

Figure 9. Test doll

temperature. Overlaid thermal and RGB images of test system is given in Figure 9.

The final point considered in the design is the emissivity difference between the plastic of the doll and the human body, whose emissivity levels are respectively 0.95 and 0.99 . In order to minimize the emissivity difference, a piece of black tape is attached on the forehead of the doll, both to cover the thermocouple and to measure the accurate temperature over the region.

\section{RESULTS}

A set of observations is done to evaluate the performance of the design on the test system. Reliability of the software architecture, multimodal registration and temperature model constitutes the necessary conditions to demonstrate a successful proof-of-concept.

\section{A. Validity of Temperature Model}

The main expectation of the temperature model is to comply with the reference graphic provided in the datasheet of the FLIR Lepton thermal cameras, as seen in the Figure 10. The graph provided by the dataset illustrates the hypothetical case of a radiometry-enabled thermal camera, that is able to provide real scene temperatures directly to

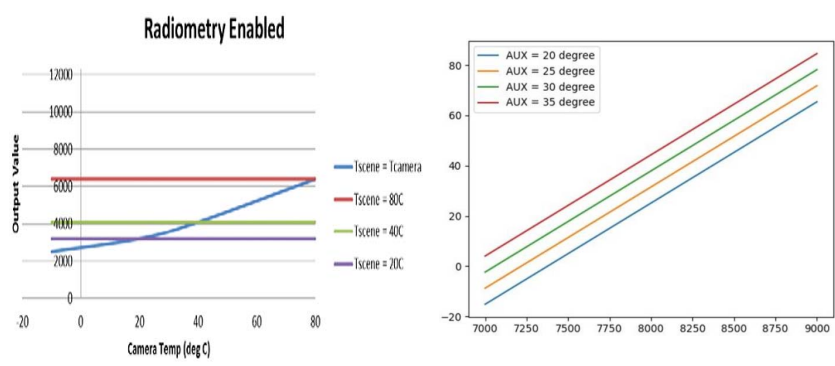

Figure 10. (Left) Hypothetical Illustration of Camera Output vs. Camera Temperature in Radiometry-enabled Mode taken from [15], and (right) calibrated model using Elastic Net regularization. On the right graph, $\mathrm{x}$-axis stands for the output value and the y-axis is equal to the Tscene of the graph from the datasheet.

the device. Since the thermal camera in the proposed design lacks this feature, the temperature model has to reflect similar properties with the radiometry-enabled case.

When observed, it is evident that the regression model behaves as expected, and it also delivers accuracy of as high as 0.4 degrees according to the test results. The temperature model can be deemed valid based on these results, yet it is important to note that the obtained accuracy is not in a medical level, and the prototype should not be used for such purposes unless a more accurate thermal camera is utilized instead. Furthermore, during the experiments it is observed that the casing of the thermal camera severely affects the temperature model, therefore the Equation 1 may not be valid for a similar system.

\section{B. Performance of Multiprocessing Architecture}

Resources of an embedded system with the purpose of home-use is limited. Furthermore, we are also limited by cost constraints as at this price point it is not possible to use an expensive thermal camera or powerful processing units. Thus, we proposed a multiprocessing architecture built with Python, that enables the device to drive two cameras and a variety of peripherals while also handling server connection. The system caused no bottleneck and managed to do all of these tasks simultaneously with efficient handling of processes. In the end, we managed to obtain a system that can output thermalRGB fused video feed with close to 9 FPS while handling server connection and also driving a speaker, microphone and a temperature and humidity sensor.

\section{Performance of Multimodal Registration}

Alignment of the thermal and RGB frames does not have an sufficient metric to evaluate, but the reliability of the algorithm is tested on multiple conditions to ensure the adaptivity of the second-degree polynomial matrix calculation. Two different examples of such cases can be examined in Figure 11.

It is important to note here that the mapping for all studied frames provided an appropriate fit for a second degree polynomial. Therefore, it can be freely argued that the initial 

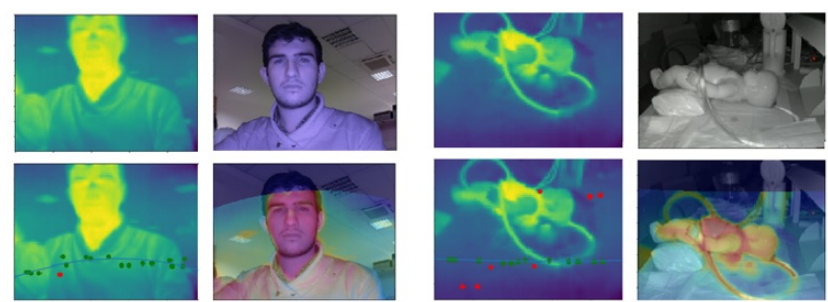

Figure 11. Two exemplary usages of frame alignment algorithm. For both images and from left to right, top to bottom: Thermal frame, RGB frame, thermal frame with polynomial approximation, and overlaid output. Red points represent the detected outliers, while green points stand for the registration vectors from the center of the image to calculate the second degree polynomial.

assumptions of polynomial warping are sufficient for the purposes of this concept. Even in the low light conditions, the illumination of IR LED's are able to fit properly onto the region of interests in the RGB frames.

As one of the downsides of the polynomial warping, the resulting overlay does not have a fixed-size rectangular bounding box. Although this problem is solvable by an inverse polynomial warping operation that will map the overlaid frame back into a rectangular area, in the current system the frames are cut to an aspect ratio of $3: 4$ for a standardized response.

\section{Feasibility of the Concept}

The feasibility of the system can be investigated by analyzing two important aspects: The reliability of the system and the cost associated with the mass-production. Although providing an exact analysis of the reliability is not possible for a prototype such as ours, we have demonstrated the successful implementation of each part in the architecture throughout this study. For the second point, we can comment on the cost while also observing the fact that the cost of manufacturing an end-product is lower than building a prototype. To give context, baby monitors which are not equipped with thermal camera for fusion and baby body temperature measurement, with similar characteristics have retail prices ranging between 75 to $130 \$$. In our cost analysis, we found the cost of building a prototype as $291 \$$. This can be interpreted as the product showing promise as a concept and can be realized, since extra features (real-time thermal video feed and baby temperature measurement) are not included in the other models. The cost is reasonable for a prototype as it is likely to go down with mass production, considering the fact that several endconsumer products are used for the prototype. Thus, the product is realizable for the current baby monitor market.

Although the algorithms and hardware design of the system is able to detect any significant temperature change and to report successfully within an error margin of $0.4^{\circ} \mathrm{C}$, it does not deliver any medical level accuracy. The human face is adaptive in terms of body temperature, and we observe that the temperature varies depending on the environment, therefore any direct measurements taken from the face using our device cannot be used as a medical reference but it can be used as an efficient tool to detect and monitor fever or hypothermia, providing comparative data over usage time through assigning a normal temperature range to the infant face, which is specific to the individual.

As a future work, it is planned to implement additional peripherals to the design in order to create a model to monitor patient health in addition to a baby monitor.

\section{CONCLUSION}

In this study, we have proposed a concept of a baby monitor that utilizes a thermal and RGB camera at the same system to provide a wider range of information to the user along with the visual and audio data. This system is designed to address fever monitoring procedures which are held manually so far and these procedures are crucial for neonatal and premature infants especially.

This project is proven to be realizable for customer usage satisfying its proof-of-concept basis. Due to current hardware limitations of the system, our device does not offer medical level accuracy and prone to be disturbed by environment conditions such as illumination, temperature and humidity. The proposed software and hardware architecture have been tested by the aforementioned test system to ensure the existence of a fully capable device, along with novel algorithms to align and process the thermal data with RGB input. Proposed system stands to be the first of its kind in terms of a fully integrated thermal and RGB sensors to provide thermal data of the person of interest, allowing parents to monitor their children easily and more extensively.

\section{ACKNOWLEDGMENT}

This study is carried out in Bilkent University. The work was supported by Vestel Electronics, TÜBİTAK 2209National/International Research Projects Fellowship Programme for Undergraduate Students 2018/2, and Bilkent University. The authors would like to thank Asst. Prof. Emine Ülkü Saritaş, Dr. Alper Kutay, Barbaros Kirişken and Mahmut Can Soydan.

\section{REFERENCES}

[1] M. S. . D. R. Laboratories, The Merck manual of diagnosis and therapy, 2018.

[2] R. B. Knobel, D. Holditch-Davis, and T. A. Schwartz, "Optimal body temperature in transitional extremely low birth weight infants using heart rate and temperature as indicators," Journal of Obstetric, Gynecologic \& Neonatal Nursing, vol. 39, no. 1, p. 314, 2010.

[3] Avent, Avent Digital Video Baby Monitor SCD630/37 Digital Video Baby Monitor, 2019. [Online]. Available: https://www.philips.ca/c-p/SCD630\{\_\}37/avent-babymonitor-digital-video-baby-monitor

[4] Babysense, Babysense Monitors, 2018. [Online]. Available: https://www.babysensellc.com/ 
[5] iBaby, iBaby Monitor M6S - The Most Advanced Wi-Fi Baby Video Monitor, 2018. [Online]. Available: https: //ibabylabs.com/shop/ibaby-monitor-m6s/

[6] N. Children, FeverSmart Temperature Monitor, 2018. [Online]. Available: https://www.nurofenforchildren.co.uk/ products/feversmart-temperature-monitor

[7] A. S. Wyckoff, Thermometer use 101. American Academy of Pediatrics, Nov 2009. [Online]. Available: https://www. aappublications.org/content/30/11/29.2

[8] P. Viola and M. Jones, "Rapid object detection using a boosted cascade of simple features," Proceedings of the 2001 IEEE Computer Society Conference on Computer Vision and Pattern Recognition. CVPR 2001.

[9] B. M. for FLIR One, Baby Monitor for FLIR ONE - Apps on Google Play, 2018. [Online]. Available: play.google.com/ store/apps/details?id=com.mtat.babymonitor.flirone

[10] Temptraq, Tempraq, 2015. [Online]. Available: https: //www.temptraq.com/Home

[11] USB Stereo Audio Adapter with $3.5 \mathrm{~mm} L / R$ Channel Speaker, NEC Avio, 2019. [Online]. Available: http://www.infrared.avio.co.jp/en/products/ir-thermo/ lineup/tvs200is $\backslash$ _tvs500is/index.html

[12] Portable Infrared Thermal Imaging Cameras, NEC Avio, 2019. [Online]. Available: https://thermal-imaging-camera. irpod.net/shop/portable-infrared-thermal-imaging-cameras/

[13] Use of Infrared to Detect Elevated Body Temperatures, FLIR, 2019. [Online]. Available: https://www.overloadsrl.it/easyUp/ file/ebola $\backslash \% 201 . p d f$

[14] FLIR A320 Industrial Automation IR Camera, FLIR, 2019. [Online]. Available: https://www.tester.co.uk/flir-a320automation-thermal-camera

[15] Lepton, FLIR, 2015. [Online]. Available: https://www.flir. com/products/lepton/

[16] Raspberry Pi Camera, Waveshare, 11 2015, rev. 1.2.1.

[17] Digital Relative Humidity and Temperature Sensor, Adafruit, 2018.

[18] USB Stereo Audio Adapter with 3.5mm L/R Channel Speaker, UGREEN, 2018. [Online]. Available: https://www.ugreen.com/ product/UGREEN $\backslash$ USB $\backslash \_S t e r e o \backslash$ Audio $\backslash \_$Adapter-en.html

[19] $P R N-202$, Piranha, 2018. [Online]. Available: http://piranha. com.tr/product.php?productid=164\\#.XJAFhigzY $2 \mathrm{w}$

[20] S. van der Walt, J. L. Schönberger, J. Nunez-Iglesias, F. Boulogne, J. D. Warner, N. Yager, E. Gouillart, T. Yu, and the scikit-image contributors, "scikit," PeerJ, vol. 2, p. e453, 6 2014. [Online]. Available: https://doi.org/10.7717/peerj.453

[21] L. Chen, N. Shashidhar, and Q. Liu, "Scalable secure mjpeg video streaming," 2012 26th International Conference on Advanced Information Networking and Applications Workshops, 2012.

[22] A. Chambolle, "An algorithm for total variation minimization and applications," Journal of Mathematical Imaging and Vision, vol. 20, p. 8997, 2004.

[23] R. Fletcher and M. J. D. Powell, "A rapidly convergent descent method for minimization," The Computer Journal, vol. 6, no. 2, p. $163168,1963$.

[24] M. Guizar-Sicairos, S. T. Thurman, and J. R. Fienup, "Efficient subpixel image registration algorithms," Optics Letters, vol. 33, no. 2, p. 156, 2008.

[25] J. L. Devore, Probability and statistics for engineering and the sciences. Cengage Learning, 2017.
[26] B. Zitov and J. Flusser, "Image registration methods: a survey," Image and Vision Computing, vol. 21, no. 11, p. 9771000, 2003.

[27] Y. Hu, M. Modat, E. Gibson, W. Li, N. Ghavami, E. Bonmati, G. Wang, S. Bandula, C. M. Moore, M. Emberton, and et al., "Weakly-supervised convolutional neural networks for multimodal image registration," Medical Image Analysis, vol. 49, p. 113, 2018.

[28] Y. Lu, K. Gao, T. Zhang, and T. Xu, "A novel image registration approach via combining local features and geometric invariants," Plos One, vol. 13, no. 1, 2018.

[29] M. E. Akbiyik, "Unsupervised multimodal image registration by polynomial warping over correlation-maximizing shifts," Journal of Physics: Conference Series, vol. 1438, p. 012020, jan 2020. [Online]. Available: https://doi.org/10.1088/1742$6596 / 1438 / 1 / 012020$

[30] H. Zou and T. Hastie, "Regularization and variable selection via the elastic net," Journal of the Royal Statistical Society, Series B, vol. 67, pp. 301-320, 2005.

[31] O. Zara, "Sql designer," Sep 2011.

[32] Programmable Resolution 1-Wire Digital Thermometer, Maxim Integrated, 9 2018, rev. 5. 